\title{
REFERENCES
}

Lehmann D. F. (1987) Primidone crystalluria following overdose-a report of a case and an analysis of the 3 literature. Medical Toxicology 2, 383-7.

Morley D. \& Wynne N. A. (1957) Acute primidone poisoning in a child. British Medical fournal 1, 90.

\section{Serum potassium response to nebulized salbutamol}

Sir

If the adrenalin-mediated side-effects of nebulized Salbutamol were entirely deter- $\stackrel{\varpi}{\varpi}$ mined by its systemic absorption, one would have expected to have demonstrated a $\overrightarrow{0}$ significant correlation between pre-treatment, peak expiratory flow rate (PEFR) and $\vec{\omega}$ hypokalaemia. In our study, however, patients 5,17 and 19 failed to respond in a manner that was in keeping with this postulation. Similarly, the quoted discrepancies $\stackrel{\Phi}{3}$ noted by Ind $e t a l$. and Zimmerman indicate that there is more to the problem than mere $\dot{\sigma}$ absorption, important as this factor might be.

My own feelings are that in a given patient, the hypokalaemic response to Salbutamol $\underset{\infty}{\infty}$ will also be determined by the pre-existing $\mathrm{pH}, \mathrm{pO} 2, \mathrm{pCO} 2$, and previous medication (corticosteroids, Theophylline, etc). Interestingly, our study indicates that if an attack 3 of asthma is severe enough to induce respiratory acidosis, nebulized Salbutamol does not induce hypokalaemia.

There is now a trend towards administering intravenous potassium supplements $\frac{\mathbb{1}}{3}$ concurrently with intravenous Salbutamol. With regards to nebulized Salbutamof however, there are two paths to consider. Once can either begin to use potassium $\overrightarrow{0}$ supplements routinely, or one can try and identify specific patient subgroups who are risk of developing hypokalaemia. Hopefully, we shall soon be treated to a good qualie study, based on the latter more logical approach.

\section{J. DACRUZ}

Department of Accident and Emergency,

Torbay Hospital,

Torquay,

South Devon, England

\section{Trauma audit}

Sir

May I comment on some of the logical and statistical aspects of the interesting series of $\bigcirc$ papers on trauma audit in the June issue of Archives of Emergency Medicine.

The concept of Probability of survival or of death is crucial to these studies. In the numerical form of say $P s=0 \cdot 6$ derived from TRISS or from ISS/Age, the implication is $\mathcal{O}$ that of 10 such cases it is estimated that 6 will survive and 4 will die. The particular $N$ patient may be one of the ' 4 ' or one of the ' 6 '. It follows that the use of the Ps value in $\underset{\mathcal{W}}{\mathcal{W}}$ respect of a single case is quite limited. It might conceivably be used like the 'form' of horses as the basis for laying bets but it does not mean that this case is 'expected' to live; 0 nor, if the Ps value were $\mathbf{0 . 4}$, would it mean that he is 'expected' to die. Not all races are won by odds-on favourites! 
The most useful application of the 'probability' approach is for assessing, in respect of mortality, the overall performance of say an accident service or a certain regime of treatment. For this it is essential that the probability values of all patients whether surviving or dead are taken into account. A simple guide as to whether the target is being reached is to add all these values. In the case of Ps this will give the expected number of survivors; this can then be compared with the observed number. The ' $Z$ ' statistic of Boyd et al., (1987) gives a method for testing significance ('observed' minus 'expected' is the numerator in the ' $Z$ ' formula). The paper of Montague $\&$ Brooks, ( $p$. 116 of the June issue), gives a good example of the application of this method. Addition of ' $P$ ' values of survivors and deaths from the ISS/Age chart in the study by Underhill \& Finlayson, (p. 90), would also have given a guide to overall performance, in this case by the number of expected and observed deaths.

Probability values for deaths can be used for classifying them into a priori high and low-risk groups as was done by Underhill \& Finlayson. This helps to structure an audit of deaths but cannot be used to assess overall performance. There may, for instance, have been outstanding success in high-risk patients but this is ignored by concentrating on the deaths. The same considerations apply to the paper by Gordon et al., (p. 107), where again only deaths are considered-this time classified by TRISS and Ps.

Attempts have been made to judge performance by taking the average (either mean or median) of the ISS scores of cases which die. The plausable suggestion is then made that a high value of this 'Mean Lethal Score' (MLS) implies a good result and a low one correspondingly poorer. The letter from Court-brown in British Medical Journal. January 141989 p.115 illustrates the difficulties of interpreting this index. As a guide to overall performance it is seriously fallacious. Firstly, like all methods based only on deaths, it ignores 'successes' among survivors and secondly, it is greatly affected by the 'mix' of severities in the series studied. Brief reflection will show that if two series are equally well treated and meet the survival 'target' but if one has high proportion of severe cases and the other mostly has moderate cases the first will unjustly score a higher MLS than the second. An even more sinister implication is that the way to get a high and therefore favourable MLS is to admit more very severe cases and then let them die! To strive successfuly for their survival might well lower the MLS and indicate poorer performance.

To summarize-severity indices can provide a good method for scaling trauma severity. The mortality probabilities derived from them offer an assessment, from the survival point of view, of results in a series of cases if, and only the values of both surviving and fatal cases are taken into account. The probability value of an individual case has very limited valid use; the temptation to consider that a Ps of more than 0.5 means 'expected to live' or conversely that less that 0.5 implies 'expected to die' should be resisted. The short-cut of the Mean Lethal Score as a guide to general performance is seriously misleading.

\section{J. P. BULL}

MRC Burns Research Group, Birmingham Accident Hospital, Bath Row, Birmingham 